\title{
Blood-brain barrier integrity, intrathecal immunoactivation, and neuronal injury in HIV
}

\section{OPEN}

Birgitta Anesten, MD

Aylin Yilmaz, MD, $\mathrm{PhD}$

Lars Hagberg, MD, PhD

Henrik Zetterberg, MD,

$\mathrm{PhD}$

Staffan Nilsson, $\mathrm{PhD}$

Bruce J. Brew, MD, PhD

Dietmar Fuchs, PhD

Richard W. Price, MD,

$\mathrm{PhD}$

Magnus Gisslén, MD, $\mathrm{PhD}$

Correspondence to

Dr. Anesten:

birgitta.anesten@vgregion.se

Supplemental data at Neurology.org/nn

\section{ABSTRACT}

Objective: Although blood-brain barrier (BBB) impairment has been reported in HIV-infected individuals, characterization of this impairment has not been clearly defined.

Methods: BBB integrity was measured by CSF/plasma albumin ratio in this cross-sectional study of 631 HIV-infected individuals and 71 controls. We also analyzed CSF and blood HIV RNA and neopterin, CSF leukocyte count, and neurofilament light chain protein (NFL) concentrations. The HIVinfected participants included untreated neuroasymptomatic patients, patients with untreated HIV-associated dementia (HAD), and participants on suppressive antiretroviral treatment (ART).

Results: The albumin ratio was significantly increased in patients with HAD compared to all other groups. There were no significant differences between untreated neuroasymptomatic participants, treated participants, and controls. BBB integrity, however, correlated significantly with CSF leukocyte count, CSF HIV RNA, serum and CSF neopterin, and age in untreated neuroasymptomatic participants. In a multiple linear regression analysis, age, CSF neopterin, and CSF leukocyte count stood out as independent predictors of albumin ratio. A significant correlation was found between albumin ratio and CSF NFL in untreated neuroasymptomatic patients and in participants on ART. Albumin ratio, age, and CD4 cell count were confirmed as independent predictors of CSF NFL in multivariable analysis.

Conclusions: BBB disruption was mainly found in patients with HAD, where BBB damage correlated with CNS immunoactivation. Albumin ratios also correlated with CSF inflammatory markers and NFL in untreated neuroasymptomatic participants. These findings give support to the association among BBB deterioration, intrathecal immunoactivation, and neuronal injury in untreated neuroasymptomatic HIV-infected individuals. Neurol Neuroimmunol Neuroinflamm 2016;3:e300; doi: $10.1212 / \mathrm{NXI} .0000000000000300$

\section{GLOSSARY}

ART = antiretroviral therapy; $\mathbf{B B B}=$ blood-brain barrier; $\mathbf{H A D}=\mathrm{HIV}$-associated dementia; $\mathbf{N F L}=$ neurofilament light chain protein.

Impairment of the blood-brain barrier (BBB) is common in patients with HIV encephalitis and important in the pathogenesis of $\mathrm{HIV}$-associated dementia (HAD), ${ }^{1,2}$ but its characterization is incomplete.

CSF viral load and intrathecal immunoactivation, as determined by increased CSF neopterin, correlate with $\mathrm{BBB}$ impairment. ${ }^{3,4} \mathrm{CNS}$ inflammation also correlates with axonal injury, as measured by CSF concentrations of the light subunit of neurofilament protein (NFL). ${ }^{5}$ These findings indicate an association among neuroinflammation, BBB permeability, and neuronal injury, but their relationship has not been fully elucidated.

\footnotetext{
From the Department of Infectious Diseases, Institute of Biomedicine (B.A., A.Y., L.H., M.G.), and Department of Psychiatry and Neurochemistry, Institute of Neuroscience and Physiology (H.Z.), Sahlgrenska Academy, University of Gothenburg, Gothenburg, Sweden; Department of Molecular Neuroscience (H.Z.), UCL Institute of Neurology, Queen Square, London, UK; Mathematical Sciences (S.N.), Chalmers University of Technology, Gothenburg, Sweden; Departments of Neurology and HIV Medicine (B.J.B.), St Vincent's Hospital and Peter Duncan Neurosciences Unit, St Vincent's Centre for Applied Medical Research, University of New South Wales, Sydney, Australia; Division of Biological Chemistry (D.F.), Biocenter, Innsbruck Medical University, Innsbruck, Austria; and Department of Neurology (R.W.P.), University of California San Francisco.

Funding information and disclosures are provided at the end of the article. Go to Neurology.org/nn for full disclosure forms. The Article Processing Charge was paid by the authors.

This is an open access article distributed under the terms of the Creative Commons Attribution-NonCommercial-NoDerivatives License 4.0 (CC BY-NC-ND), which permits downloading and sharing the work provided it is properly cited. The work cannot be changed in any way or used commercially without permission from the journal.
} 
Initiation of antiretroviral therapy (ART) reduces CSF HIV RNA ${ }^{6}$ and various markers of inflammation and neuronal injury., ${ }^{5,7}$ In a study with 38 neuroasymptomatic individuals, ${ }^{8}$ only $5 \%$ (2/38) had elevated albumin ratios prior to ART initiation: both normalized on treatment. In a recent cross-sectional study, $38.6 \%$ of untreated and $24.6 \%$ of treated participants had signs of $\mathrm{BBB}$ impairment. ${ }^{?}$

The purpose of this study was to define the prevalence of BBB disruption in a large number of treated and untreated HIV-infected individuals at different stages of infection and CNS injury. To test the hypothesis that $\mathrm{BBB}$ function is associated with neuroinflammation and CNS injury in HIV, we analyzed associations between albumin ratios and markers of CNS immunoactivation and neuronal injury in samples from predefined patient groups representing essential stages of HIV disease progression and viral suppression.

METHODS Patients. In this retrospective cross-sectional study, we analyzed archived CSF samples from 631 HIVinfected adults and 71 HIV-negative controls from 3 centers (Gothenburg, Sweden; San Francisco, CA; and Sydney, Australia). Neurocognitive testing was not routinely performed on participants without any neurologic symptoms or complaints. All participants were clinically assessed for neurologic and neurocognitive symptoms. Those without neurologic complaints or signs at inclusion are referred to as neuroasymptomatic participants. HIV-infected participants were divided into 7 HIV-infected groups: untreated neuroasymptomatic participants, stratified into 4 groups according to $\mathrm{CD} 4$ level; untreated participants with $\mathrm{HAD}$, stratified into 2 groups according to severity of dementia; and participants on ART (table 1). The neuroasymptomatic untreated groups were as follows:
(1) neuroasymptomatic with CD4+ T-cell count $>350$ ( $\mathrm{n}=$ 125); (2) neuroasymptomatic with CD4+ T-cell count 200349 ( $\mathrm{n}=117$ ); (3) neuroasymptomatic with CD4+ T-cell count 50-199 ( $\mathrm{n}=102$ ); and (4) neuroasymptomatic with CD4+ T-cell count $<50(\mathrm{n}=71)$ cells $/ \mu \mathrm{L} .{ }^{5}$ The 2 groups of patients with HAD were (5) untreated patients with HAD stage $1^{10}(\mathrm{n}=24)$ and $(6)$ untreated patients with HAD stage $2-4(\mathrm{n}=33)$. The final group (7) consisted of participants on ART with plasma HIV RNA $<50$ copies $/ \mathrm{mL}$ for $\geq 6$ months ( $n=159$ ). Individuals with CNS opportunistic or other infections, CNS neoplasms, acute cranial nerve paresis, or neurologic diseases with CNS involvement, were excluded. The diagnosis of HAD was based on Centers for Disease Control and Prevention and American Academy of Neurology Task Force Criteria using standard laboratory, neuropsychological testing, and clinical evaluations. ${ }^{11,12}$ Most of the participants were studied before publication of the formal Frascati criteria $^{11}$ and were diagnosed with AIDS dementia complex stages $1-4^{10}$ : they met the functional criteria for the Frascati diagnosis of $\mathrm{HAD}$ without the requisite extensive formal neuropsychological assessment.

Standard protocol approvals, registrations, and patient consents. CSF samples were obtained under the auspices of research protocols approved by the institutional review boards of each of the study sites and in accordance with the Helsinki Declaration, either within the context of studies of the natural history of HIV infection (neuroasymptomatic participants and participants on ART) or during diagnostic evaluations (patients with HAD). All participants gave informed consent, and if their capacity to provide consent was questioned, consent was also obtained from those with power of attorney.

Methods. Quantitative determination of CSF and plasma albumin was performed by nephelometry (Behring Nephelometer Analyser, Behringwerke AG, Marburg, Germany). The albumin ratio was calculated as CSF albumin $(\mathrm{mg} / \mathrm{L}) /$ plasma albumin $(\mathrm{g} / \mathrm{L})$ and used to evaluate BBB function. Reference values were $<6.8$ for individuals younger than 45 years and $<10.2$ for individuals $\geq 45$ years of age. ${ }^{13}$ Some CSF samples had been frozen, but never refrozen: regular controls with analyses of biological markers have confirmed compatibility between fresh and frozen samples.

CSF neopterin was analyzed using a commercially available immunoassay (BRAHMS, Berlin, Germany) according to the manufacturer's instructions. Normal CSF neopterin reference

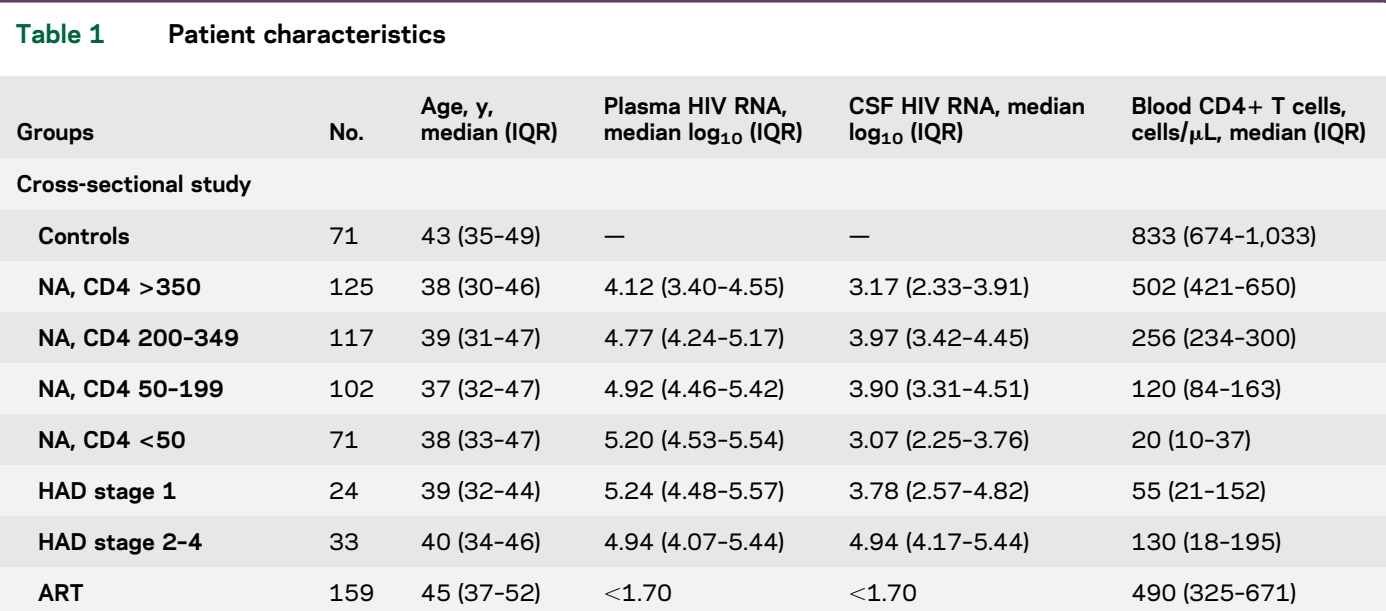

Abbreviations: ART $=$ on antiretroviral treatment with P-RNA $<50$ copies $/ \mathrm{mL} ; \mathrm{HAD}=\mathrm{HIV}$-associated dementia (untreated); $\mathrm{IQR}=$ interquartile range; $\mathrm{NA}=$ neuroasymptomatic (untreated). 
Figure $1 \quad$ CSF/plasma albumin ratio in the different groups of HIV-infected participants and the healthy controls

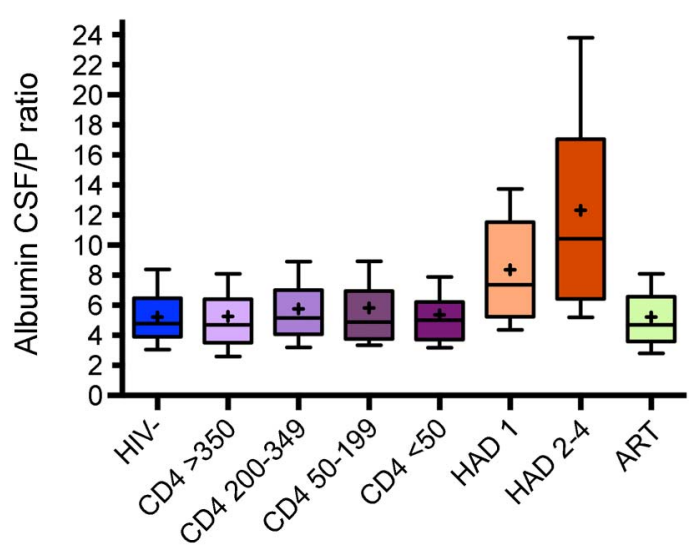

Boxes encompass interquartile ranges with median (line) and mean $(+)$, while whiskers designate 10th to 90th percentiles. Included in this cross-sectional analyses were 71 controls (HIV-); HIV-infected neuroasymptomatic participants without antiretroviral treatment (ART) stratified according to levels of blood CD4+ T-cell count $>350(n=125), 200-349(n=117), 50-199(n=$ $102)$, and $<50(n=71) ; 57$ participants with HIV-associated dementia (HAD) staged according to severity of symptoms, stage $1(n=24)$ and stage 2-4 $(n=33)$; and 159 participants on ART $\geq 6$ months and plasma HIV RNA $<50$ copies $/ \mathrm{mL}$. Albumin ratios were significantly higher in patients with HAD (stage 1-4 pooled) compared to all other groups $(p<0.001)$.

value was $<5.8 \mathrm{nmol} / \mathrm{L} .{ }^{14} \mathrm{CSF}$ concentrations of NFL were measured by an enzymatic 2-site quantitative immunoassay (NF-light ELISA kit; UmanDiagnostics AB, Umeå, Sweden). The upper normal reference limits of CSF NFL were $<380$ (18-30 years), $<560$ (30-39 years), $<890$ (40-59 years), and $<1,850 \mathrm{ng} / \mathrm{L}$ ( $>59$ years).

HIV RNA levels in blood and CSF were quantified by the Roche Amplicor Monitor version 1.5, Abbott RealTime HIV-1 assay (Abbott Laboratories, Abbott Park, IL), or Roche Taqman assay version 1 or 2 (Hoffman La-Roche, Basel, Switzerland). The lower limit of detection varied between 20 and $50\left(1.70 \log _{10}\right)$ HIV RNA copies/mL in the different assays. Blood CD4+ T-cell counts, CSF leukocytes, and CSF/blood albumin measurements were performed in the local clinical laboratories.

Statistical analysis. Descriptive statistics were performed using Prism (version 6.0, GraphPad, La Jolla, CA) or SPSS (version 20, IBM, Armonk, NY) software. All continuous variables except age and leukocyte count were $\log _{10}$ transformed to reduce skewness. Differences between multiple groups were tested with ordinary 1-way analysis of variance with Tukey multiple comparisons post hoc test; Student $t$ test was used for comparisons between 2 groups. Associations were analyzed with Pearson correlation coefficients. Predictors of albumin ratio and CSF NFL were analyzed by multiple linear regression analysis with forward selection.

RESULTS A total of 631 HIV-positive patients and 71 HIV-negative controls were studied (table 1). Participants with HAD (stage 1-4 pooled) had significantly higher albumin ratios compared with all the other groups $(p<0.001)$ (figure 1 and table 2). The highest albumin ratios were found in patients with the most severe HAD (stages 2-4). Albumin ratios were elevated above age-dependent reference values ${ }^{13}$ in 76\% (25/33) of patients with HAD stage $2-4$ and in $58 \%(14 / 24)$ of patients with HAD stage 1 . Sixtyeight percent (39/57) of HAD participants had elevated albumin ratios compared with 16\% (66/415) of untreated neuroasymptomatic participants. There were no statistically significant differences in albumin ratios between untreated neuroasymptomatic patients, participants on ART, and HIV-negative controls. Similarly, there were no significant differences in albumin ratios among untreated neuroasymptomatic participants with different CD4 cell strata: $12 \%$ with CD $4+$ T-cell count $>350,20 \%$ with CD4 count 200-349, 17\% with CD4 count 50-199, and 15\% with CD 4 count $<50$ cells $/ \mu \mathrm{L}$ had elevated albumin ratios. Eleven percent of HIV-negative controls and $12 \%$ of participants on suppressive ART had elevated albumin ratios.

In order to further characterize BBB impairment, in neuroasymptomatic participants, we examined the

\begin{tabular}{|c|c|c|c|c|c|c|}
\hline \multirow{2}{*}{$\begin{array}{l}\text { Table } 2 \\
\text { Groups }\end{array}$} & \multicolumn{6}{|c|}{ Ibumin ratio, inflammatory markers, and CSF NFL in the different groups of HIV-infected participants and the healthy controls } \\
\hline & No. & $\begin{array}{l}\text { Albumin ratio, } \\
\text { median (IQR) }\end{array}$ & $\begin{array}{l}\text { S-neopterin, nmol/L, } \\
\text { median (IQR) }\end{array}$ & $\begin{array}{l}\text { CSF-neopterin, } \\
\text { nmol/L, median (IQR) }\end{array}$ & $\begin{array}{l}\text { CSF WBC, cells/ } \mu \mathrm{L}, \\
\text { median (IQR) }\end{array}$ & $\begin{array}{l}\text { CSF NFL, ng/L, } \\
\text { median (IQR) }\end{array}$ \\
\hline NA, CD4 >350 & 125 & $4.70(3.53-6.40)$ & $11.1(8.4-17.9)$ & $11.2(8.1-17.7)$ & $5(3-9)$ & $440(272-580)$ \\
\hline NA, CD4 200-349 & 117 & $5.15(4.09-7.00)$ & $18.2(11.2-23.2)$ & $18.3(12.0-26.4)$ & $6(3-13)$ & $395(228-640)$ \\
\hline NA, CD4 <50 & 71 & $5.00(3.76-6.21)$ & $23.5(16.5-37.8)$ & $22.0(11.5-35.0)$ & $1(0-2)$ & $801(477-2,380)$ \\
\hline HAD stage 1 & 24 & $7.37(5.26-11.31)$ & $22.5(17.0-33.8)$ & $29.4(24.6-61.5)$ & $3(2-9)$ & $11,400(5,224-38,225)$ \\
\hline HAD stage $2-4$ & 33 & $10.42(7.20-17.00)$ & $26.4(18.2-38.9)$ & 75.0 (34.7-127.5) & $11(4-26)$ & $30,825(25,898-35,753)$ \\
\hline ART & 159 & $4.70(3.61-6.52)$ & $8.4(5.7-11.0)$ & $6.7(4.8-9.3)$ & $0(0-2)$ & $454(289-673)$ \\
\hline
\end{tabular}

Abbreviations: ART = on antiretroviral treatment with P-RNA $<50$ copies $/ \mathrm{mL} ; \mathrm{HAD}=\mathrm{HIV}$-associated dementia (untreated); IQR $=$ interquartile range; $\mathrm{NA}=$ neuroasymptomatic (untreated); NFL = neurofilament light chain protein; WBC = white blood cells. 
CSF biomarker profiles in participants with increased as opposed to normal albumin ratios with respect to age-dependent reference values.

Neuroasymptomatic participants with increased albumin ratios had higher CSF neopterin $(p<$ $0.05)$ and CSF NFL levels $(p<0.01)$. No difference was found in CSF HIV RNA between those groups (figure 2). Patients on suppressive ART and HIVnegative controls with increased albumin ratios had similar levels of CSF neopterin and CSF NFL as participants with normal ratio (not shown).

Correlations of CSF biomarkers and albumin ratio. In untreated neuroasymptomatic participants, albumin ratios correlated with CSF leukocyte count $(r=0.25, p<0.001)$, CSF HIV RNA levels $(r=0.15, p<0.01)$, blood neopterin $(r=0.21$, $p<0.001)$, CSF neopterin $(r=0.26, p<0.001)$, and age $(r=0.24, p<0.001)$. Similar correlations with age $(r=0.27, p=0.001)$ and CSF leukocytes $(r=0.16, p=0.05)$ were found in virally suppressed participants. In HIV-negative controls, only age correlated significantly with albumin ratio $(r=0.26, p<0.05)$. No significant correlations were found between CSF neopterin and albumin ratio in participants on suppressive ART or in HIV-negative controls. There was no significant correlation between CD4 nadir and albumin ratio in virally suppressed participants. While all participants on ART had plasma HIV RNA levels $<50$ copies $/ \mathrm{mL}$ as part of the inclusion criteria, 5 out of 159 treated participants $(3.1 \%)$ had detectable HIV RNA levels in the CSF, ranging from 50 to 145 copies/mL.

In a multiple linear regression analysis, age, CSF neopterin, and CSF leukocyte count stood out as independent predictors of albumin ratios in neuroasymptomatic participants (table 3 ).

Correlations between albumin ratio and CSF NFL. A significant correlation was found between the albumin ratio and CSF NFL concentration in untreated neuroasymptomatic participants $(r=0.32, p<0.001)$.

Albumin ratio was confirmed as an independent predictor of CSF NFL together with age and CD4 cell count in a multiple linear regression analysis. By contrast, CSF HIV RNA and CSF neopterin were not found to be significant predictors (table 4).

Albumin ratios and CSF NFL levels were also correlated in participants on ART $(r=0.44, p<0.001)$ and albumin ratios were, together with age, confirmed as independent predictors of CSF NFL in multivariable analysis (data not shown). Likewise, a significant correlation was found between albumin ratios and CSF NFL concentrations in HIV-negative controls $(r=0.30, p<0.05)$. CSF NFL results were
Figure 2 CSF biomarkers in HIV-infected untreated neuroasymptomatic participants with damaged and normal blood-brain barrier (BBB)

\section{A. CSF HIV RNA}

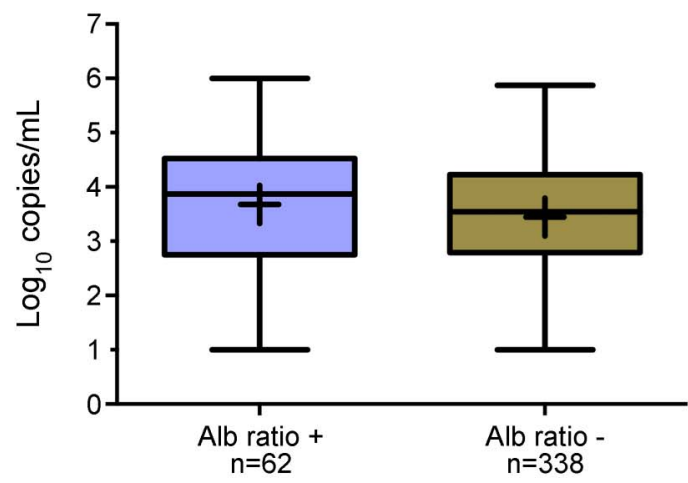

B. CSF neopterin

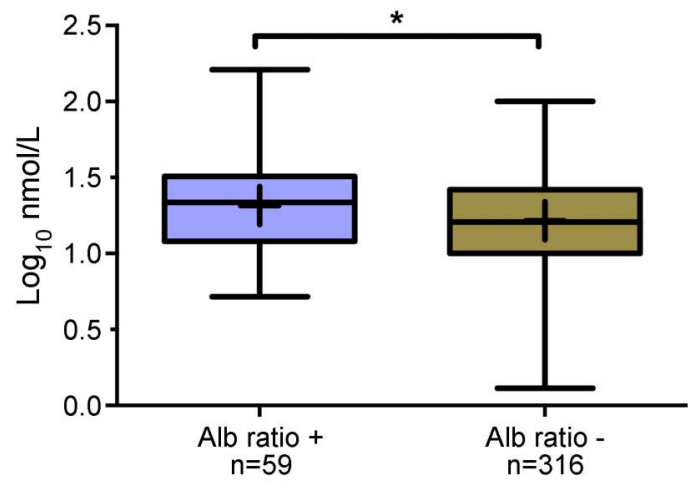

C. CSF NFL

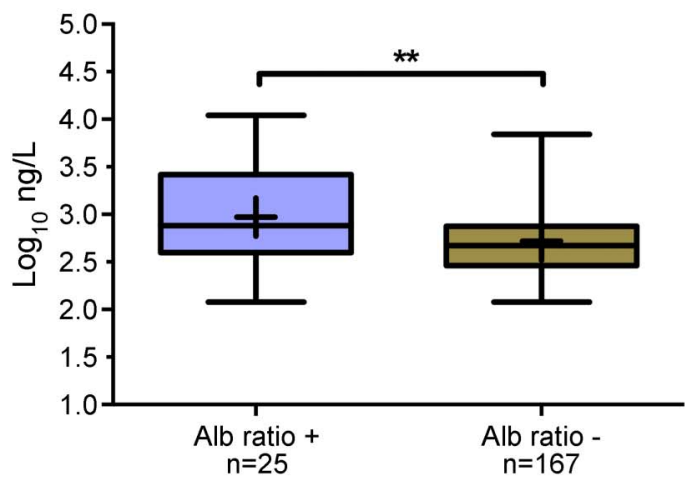

Comparisons of CSF biomarkers in HIV-infected untreated neuroasymptomatic participants with impaired (Alb ratio + ) and normal (Alb ratio -) BBB function with respect to age-dependent reference values. CSF HIV RNA levels did not differ significantly between the groups (A) whereas CSF neopterin $(p<0.05, B)$ and CSF neurofilament light chain protein (NFL) $(p<0.01, C)$ were higher in participants with impaired BBB compared with those with normal BBB.

missing in the majority of patients with HAD because of shortage of samples.

DISCUSSION BBB impairment is considered a key event in CNS injury in HIV infection. To date, this is the largest study that has characterized the BBB in 


\begin{tabular}{|c|c|c|c|c|}
\hline \multirow{3}{*}{$\begin{array}{l}\text { Table } 3 \\
\text { Predictor }\end{array}$} & \multicolumn{4}{|c|}{$\begin{array}{l}\text { Univariable correlation (left columns) } \\
\text { and multiple linear regression (right } \\
\text { columns) determining predictors of } \\
\text { CSF/plasma albumin ratio in } 415 \mathrm{HIV}- \\
\text { infected neuroasymptomatic patients } \\
\text { without antiretroviral treatment }\end{array}$} \\
\hline & \multicolumn{2}{|c|}{ Univariable } & \multicolumn{2}{|c|}{ Multivariable } \\
\hline & Std $B(r)$ & $p$ Value & Std $B_{\text {adj }}$ & $p$ Value \\
\hline Age & 0.242 & $<0.001$ & 0.222 & $<0.001$ \\
\hline Blood CD4 & 0.010 & 0.84 & & \\
\hline Blood CD8 & -0.009 & 0.85 & & \\
\hline CSF WBC & 0.245 & $<0.001$ & 0.132 & 0.015 \\
\hline P HIV RNA & 0.097 & 0.055 & & \\
\hline CSF HIV RNA & 0.153 & 0.002 & & \\
\hline S neopterin & 0.208 & $<0.001$ & & \\
\hline CSF neopterin & 0.259 & $<0.001$ & 0.187 & 0.001 \\
\hline
\end{tabular}

Abbreviation: $\mathrm{CSF}=$ cerebrospinal fluid; $\mathrm{P}=$ plasma; $S=$ serum; Std $B=$ standardized beta coefficient; Std $\beta_{\text {adj }}=$ adjusted standardized beta coefficient; WBC = white blood cells.

treated and untreated HIV-infected individuals at different stages of infection, and with varying degrees of neurocognitive impairment. A majority of the participants with HAD (68\%) had elevated albumin ratios, compared with $16 \%$ of untreated neuroasymptomatic individuals. This is broadly consistent with previous smaller studies, where the prevalence of BBB dysfunction has been shown to increase as the HIV infection progresses., ${ }^{315-17}$ In contrast to earlier publications that have been fairly small or based on case reports, this study extends the characterization of $\mathrm{BBB}$ dysfunction and its relationship to neuroinflammation and CNS injury

\begin{tabular}{|c|c|c|c|c|}
\hline \multirow{3}{*}{$\begin{array}{l}\text { Table } 4 \\
\text { Predictor }\end{array}$} & \multicolumn{4}{|c|}{$\begin{array}{l}\text { Univariable correlation (left columns) } \\
\text { and multiple linear regression (right } \\
\text { columns) determining predictors of CSF } \\
\text { NFL in } 415 \text { HIV-infected } \\
\text { neuroasymptomatic patients without } \\
\text { antiretroviral treatment }\end{array}$} \\
\hline & \multicolumn{2}{|c|}{ Univariable } & \multicolumn{2}{|c|}{ Multivariable } \\
\hline & Std $B(r)$ & p Value & Std $B_{\text {adj }}$ & $p$ Value \\
\hline Age & 0.365 & $<0.001$ & 0.341 & $<0.001$ \\
\hline Blood CD4 & -0.326 & $<0.001$ & -0.392 & $<0.001$ \\
\hline CSF WBC & -0.090 & 0.22 & & \\
\hline CSF HIV RNA & -0.070 & 0.34 & & \\
\hline CSF neopterin & 0.200 & 0.010 & & \\
\hline $\begin{array}{l}\text { CSF/P albumin } \\
\text { ratio }\end{array}$ & 0.315 & $<0.001$ & 0.264 & $<0.001$ \\
\hline
\end{tabular}

Abbreviations: $\mathrm{P}=$ plasma; $\mathrm{NFL}=$ neurofilament light chain protein; Std $B=$ standardized beta coefficient; Std $\beta_{\text {adj }}=$ adjusted standardized beta coefficient; $W B C=$ white blood cells. to this rather large cohort of thoroughly classified participants with HIV infection. This is a major strength of the study and makes the results more reliable.

Although most neuroasymptomatic HIV-infected participants without ART had albumin ratios within the normal range, we found a significant correlation between CSF neopterin, a marker of intrathecal immune activation, and BBB integrity in this group. CNS inflammation was, together with age, confirmed in a multivariable analysis as independent predictors of BBB impairment. Intrathecal immune activation is a general feature of HIV infection, and a persistent low-grade immune activation is often present even in antiretroviral treated patients despite several years of suppressed plasma viral loads. ${ }^{718}$ However, CSF neopterin did not correlate with albumin ratios in patients on suppressive ART, or in healthy controls, perhaps because substantial CNS inflammation is required for BBB impairment.

In addition, we found the albumin ratio to be an independent predictor of CSF NFL both in untreated and treated HIV-infected individuals, suggesting that minor impairment of the BBB might be harmful to the CNS. However, the lack of association with markers of immune activation in patients on treatment implies either an additional mechanism or possibly fixed BBB impairment as a legacy effect of pre-ART disease. Another recent study on treated HIV-infected individuals undergoing lumbar punctures for clinical reasons reported a similar association between albumin ratios and the neural protein $\mathrm{t}$-tau, but without any association with CSF neopterin. ${ }^{19} \mathrm{NFL}$ is a more sensitive marker of neural injury than $\mathrm{t}-\mathrm{tau}^{20}$ and our study corroborates the association between BBB function and CNS disturbance, extending it also to untreated and treated HIVinfected participants without neurologic symptoms.

The BBB is a semipermeable barrier surrounding the CNS. Its main function is to maintain a stable environment for the brain. It differs from other capillaries in the body in a number of ways. Most importantly, endothelial cells in the BBB are fused together by tight junctions. ${ }^{21} \mathrm{HIV}$-infected cells in the CNS and the periphery produce viral proteins such as gp120, Tat, and Nef, and inflammatory mediators including cytokines and chemokines. ${ }^{22}$ These viral and host products have the ability to affect the integrity of the $\mathrm{BBB},{ }^{23}$ leading to increased permeability of the brain endothelial cells. Impairment of the BBB could be harmful to the brain by facilitating influx of blood proteins, viral particles, and other possibly neurotoxic substances into the CNS (figure e- 1 at Neurology.org/nn). Conversely, BBB impairment may facilitate entry of antiretroviral drugs, which may be beneficial. The BBB restricts the entry of proteins from the blood to the brain and the CSF, 
resulting in markedly lower concentrations of proteins in CSF than in blood. Because albumin is exclusively synthesized in the liver, albumin detected in CSF originates from blood. The albumin ratio between CSF and plasma provides a reliable determination of $\mathrm{BBB}$ function, ${ }^{13,24}$ and is the most commonly used method to examine this.

Myelinated axons consist of a major structural element NFL, the light subunit of the neurofilament protein. Previous studies have confirmed CSF NFL concentrations as a reliable marker of CNS axonal injury in various neurodegenerative diseases ${ }^{25}$ including CNS injury associated with HIV infection.5,26 Increased CSF NFL is a consistent finding in patients with HAD, but can also be found in some HIVinfected patients without neurologic symptoms, mainly in individuals with low CD4+ T-cell counts. Our study confirms immunosuppression and age as predictors of CSF NFL in multivariable analysis. In addition, we found that impairment of the $\mathrm{BBB}$ was an independent predictor of axonal injury. A significant correlation was found between the albumin ratio and CSF NFL in both untreated and treated individuals, supporting the hypothesis that increased BBB permeability may be linked with neuronal injury in HIV infection. Neurotoxic substances and inflammation may cause damage to neuroglial cells further amplifying BBB breakdown, eventually leading to clinically significant neurocognitive deficits.

In agreement with previous smaller studies, ${ }^{3}$ we found no correlation between $\mathrm{CD} 4+\mathrm{T}$-cell count and $\mathrm{BBB}$ dysfunction. Another recent study found that increased $\mathrm{BBB}$ permeability was more common in individuals on ART with low CD4 nadir compared with those with high CD4 nadir. ${ }^{\text {W We could not }}$ confirm this finding in our study, where no correlation was found between CD4 nadir and the albumin ratio in patients on suppressive ART. In contrast to the abovementioned study, all treated participants in our study were virologically suppressed, which might explain the diverse results. Furthermore, in the current study, participants were asymptomatic, with lumbar punctures performed within clinical study protocols and not for clinical reasons.

Also in agreement with previous studies, ${ }^{9}$ we did not find any difference in the albumin ratio between neuroasymptomatic HIV-infected participants and patients on ART. However, those were cross-sectional studies and the data are sparse on BBB integrity changes following initiation of ART. ${ }^{8}$ Neither has the effect of ART on BBB integrity in patients with $\mathrm{HAD}$ and increased albumin ratios been carefully elucidated. Smaller studies and case reports indicate that normalization of the albumin ratio, even in patients with HAD, is possible after commencement of ART. ${ }^{8,27}$
Almost one-fifth of untreated neuroasymptomatic participants, some of them with preserved immune systems, had signs of BBB impairment. Patients in the neuroasymptomatic groups had neither neurologic nor neurocognitive symptoms, signs, or complaints, but as neuropsychological testing was not performed on all participants, we cannot exclude the possibility that some had asymptomatic neurocognitive impairment. It is not known if asymptomatic patients with elevated albumin ratios have an increased risk of developing symptomatic neurocognitive deficits in the future. It is notable that the proportion of asymptomatic untreated patients with impaired BBB had significantly higher levels of both CSF neopterin and CSF NFL compared with patients without BBB injury, while no such difference was found in participants on suppressive ART or in healthy controls.

We have previously found that CSF NFL can predict severe neurocognitive impairment. ${ }^{28}$ Impairment of $\mathrm{BBB}$ with simultaneously raised levels of CSF neopterin and CSF NFL give further support to the association among BBB deterioration, neuroinflammation, and axonal injury in untreated HIV. Indeed, our results point to the potential utility of measurement of the albumin ratio, something which is simple and easily performed. Further, our data allow the generation of 2 important hypotheses. First, albumin ratio elevation in neuroasymptomatic patients may predict the subsequent development of HIV-associated neurocognitive disorders. Second, there is no need for antiretroviral drugs that penetrate the brain if the albumin ratio is normal. Further prospective studies can address these hypotheses.

\section{AUTHOR CONTRIBUTIONS}

M.G. originated the idea and designed and supervised the study. M.G., A.Y., L.H., B.B., and RW.P. recruited the participants. D.F. and H.Z. performed the biochemical analyses. B.A. and M.G. performed acquisition, analysis, and interpretation of data. B.A., M.G., and S.N. performed the statistics. B.A., A.Y., and M.G. wrote the article. All the authors contributed to manuscript preparation.

\section{STUDY FUNDING}

Supported by the Swedish Research Council (K2011-58P-20931-01-4, 2013-2546), the Sahlgrenska University Hospital (ALFGBG-430271, ALFGBG-441051), the Knut and Alice Wallenberg Foundation, and the NIH (R01MH62701, R21MH096619, R21NS069219 and UL1 TR000004).

\section{DISCLOSURE}

B. Anesten and A. Yilmaz report no disclosures. L. Hagberg serves as an associate editor for Infectious Diseases. H. Zetterberg served as an associate editor for Journal of Alzheimer's Disease, Alzheimer's \& Dementia, and $D A D M$; is cofounder of Brain Biomarker Solutions in Gothenburg AB; and received research support from The Swedish Research Council, Swedish State Support for Clinical Research, VINNOVA, The Knut and Alice Wallenberg Foundation, The European Research Council, and Frimurarestiftelsen. S. Nilsson reports no disclosures. B. Brew served on the scientific advisory board for Biogen Idec ViiV, received speaker 
honoraria from Biogen Idec, holds a patent for Monoclonal antibody for quinolinic acid as part of test kit for monitoring MS severity and progression, received publishing royalties from Oxford University Press and Cambridge University Press, and received research support from Biogen Idec and NIH NHMRC. D. Fuchs is Chief Editor for Pteridines. R.W. Price received travel funding and speaker honoraria from Gilead and speaker honoraria from Abbvie, consulted for Merck \& Co., and received research support from Kinemed, NIDA, NIMH, NIAID, and National Institute of Neurological Disorders and Stroke. M. Gisslen served on the scientific advisory board for Gilead Sciences, BMS, Janssen, and MSD; received speaker honoraria from Gilead, BMS, and Janssen; was editor for HIV \& Virology News and AIDS Research and Therapy; and received research support from Gilead and Janssen. Go to Neurology.org/nn for full disclosure forms.

Received March 24, 2016. Accepted in final form September 29, 2016.

\section{REFERENCES}

1. Valcour V, Chalermchai T, Sailasuta N, et al. Central nervous system viral invasion and inflammation during acute HIV infection. J Infect Dis 2012;206:275-282.

2. Gisslen M, Chiodi F, Fuchs D, et al. Markers of immune stimulation in the cerebrospinal fluid during HIV infection: a longitudinal study. Scand J Infect Dis 1994;26: 523-533.

3. Andersson LM, Hagberg L, Fuchs D, Svennerholm B, Gisslen M. Increased blood-brain barrier permeability in neuro-asymptomatic HIV-1-infected individuals: correlation with cerebrospinal fluid HIV-1 RNA and neopterin levels. J Neurovirol 2001;7:542-547.

4. Persidsky Y, Zheng J, Miller D, Gendelman HE. Mononuclear phagocytes mediate blood-brain barrier compromise and neuronal injury during HIV-1-associated dementia. J Leukoc Biol 2000;68:413-422.

5. Jessen Krut J, Mellberg T, Price RW, et al. Biomarker evidence of axonal injury in neuroasymptomatic HIV-1 patients. PLoS One 2014;9:e88591.

6. Mellgren A, Antinori A, Cinque P, et al. Cerebrospinal fluid HIV-1 infection usually responds well to antiretroviral treatment. Antivir Ther 2005;10:701-707.

7. Yilmaz A, Yiannoutsos CT, Fuchs D, et al. Cerebrospinal fluid neopterin decay characteristics after initiation of antiretroviral therapy. J Neuroinflammation 2013;10:62.

8. Abdulle S, Hagberg L, Gisslen M. Effects of antiretroviral treatment on blood-brain barrier integrity and intrathecal immunoglobulin production in neuroasymptomatic HIV1-infected patients. HIV Med 2005;6:164-169.

9. Calcagno A, Alberione MC, Romito A, et al. Prevalence and predictors of blood-brain barrier damage in the HAART era. J Neurovirol 2014;20:521-525.

10. Price RW, Brew BJ. The AIDS dementia complex. J Infect Dis 1988;158:1079-1083.

11. Antinori A, Arendt G, Becker JT, et al. Updated research nosology for HIV-associated neurocognitive disorders. Neurology 2007;69:1789-1799.

12. Nomenclature and research case definitions for neurologic manifestations of human immunodeficiency virus-type 1 (HIV-1) infection: report of a Working Group of the American Academy of Neurology AIDS Task Force. Neurology 1991;41:778-785.

13. Blennow K, Fredman P, Wallin A, et al. Protein analysis in cerebrospinal fluid: II: reference values derived from healthy individuals 18-88 years of age. Eur Neurol 1993;33:129-133.

14. Hagberg L, Cinque P, Gisslen M, et al. Cerebrospinal fluid neopterin: an informative biomarker of central nervous system immune activation in HIV-1 infection. AIDS Res Ther 2010;7:15.

15. Marshall DW, Brey RL, Cahill WT, Houk RW, Zajac RA, Boswell RN. Spectrum of cerebrospinal fluid findings in various stages of human immunodeficiency virus infection. Arch Neurol 1988;45:954-958.

16. Petito CK, Cash KS. Blood-brain barrier abnormalities in the acquired immunodeficiency syndrome: immunohistochemical localization of serum proteins in postmortem brain. Ann Neurol 1992;32:658-666.

17. Power C, Kong PA, Crawford TO, et al. Cerebral white matter changes in acquired immunodeficiency syndrome dementia: alterations of the blood-brain barrier. Ann Neurol 1993;34:339-350.

18. Eden A, Price RW, Spudich S, Fuchs D, Hagberg L, Gisslen M. Immune activation of the central nervous system is still present after $>4$ years of effective highly active antiretroviral therapy. J Infect Dis 2007;196: 1779-1783.

19. Calcagno A, Atzori C, Romito A, et al. Blood brain barrier impairment is associated with cerebrospinal fluid markers of neuronal damage in HIV-positive patients. J Neurovirol 2015;22:88-92.

20. Peterson J, Gisslen M, Zetterberg H, et al. Cerebrospinal fluid (CSF) neuronal biomarkers across the spectrum of HIV infection: hierarchy of injury and detection. PLoS One 2014;9:e116081.

21. Wong AD, Ye M, Levy AF, Rothstein JD, Bergles DE, Searson PC. The blood-brain barrier: an engineering perspective. Front Neuroeng 2013;6:7.

22. Strazza M, Pirrone V, Wigdahl B, Nonnemacher MR. Breaking down the barrier: the effects of HIV-1 on the blood-brain barrier. Brain Res 2011;1399:96-115.

23. Banks WA, Robinson SM, Nath A. Permeability of the blood-brain barrier to HIV-1 Tat. Exp Neurol 2005;193: 218-227.

24. Tibbling G, Link H, Ohman S. Principles of albumin and IgG analyses in neurological disorders: I: establishment of reference values. Scand J Clin Lab Invest 1977; 37:385-390.

25. Scherling CS, Hall T, Berisha F, et al. Cerebrospinal fluid neurofilament concentration reflects disease severity in frontotemporal degeneration. Ann Neurol 2014;75: 116-126.

26. Abdulle S, Mellgren A, Brew BJ, et al. CSF neurofilament protein (NFL): a marker of active HIV-related neurodegeneration. J Neurol 2007;254:1026-1032.

27. Andersson LM, Hagberg L, Rosengren L, Fuchs D, Blennow K, Gisslen M. Normalisation of cerebrospinal fluid biomarkers parallels improvement of neurological symptoms following HAART in HIV dementia: case report. BMC Infect Dis 2006;6:141.

28. Gisslen M, Hagberg L, Brew BJ, Cinque P, Price RW, Rosengren L. Elevated cerebrospinal fluid neurofilament light protein concentrations predict the development of AIDS dementia complex. J Infect Dis 2007; 195:1774-1778. 


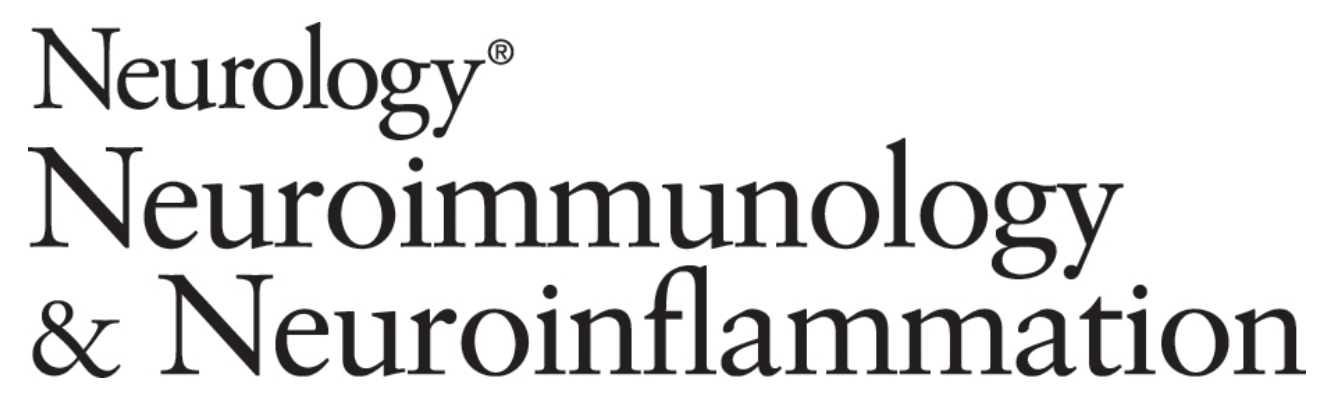

Blood-brain barrier integrity, intrathecal immunoactivation, and neuronal injury in HIV

Birgitta Anesten, Aylin Yilmaz, Lars Hagberg, et al.

Neurol Neuroimmunol Neuroinflamm 2016;3;

DOI 10.1212/NXI.0000000000000300

This information is current as of November 9,2016

Neurol Neuroimmunol Neuroinflamm is an official journal of the American Academy of Neurology.

Published since April 2014, it is an open-access, online-only, continuous publication journal. Copyright $\odot$ 2016 American Academy of Neurology. All rights reserved. Online ISSN: 2332-7812.

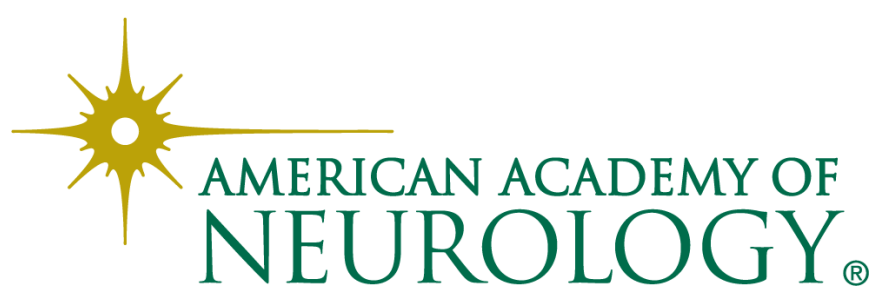




\section{Updated Information \& Services}

\section{Supplementary Material}

\section{References}

Citations

Subspecialty Collections

Permissions \& Licensing

\section{Reprints}

including high resolution figures, can be found at: http://nn.neurology.org/content/3/6/e300.full.html

Supplementary material can be found at: http://nn.neurology.org/content/suppl/2016/11/09/3.6.e300.DC1

This article cites 28 articles, 0 of which you can access for free at: http://nn.neurology.org/content/3/6/e300.full.html\#\#ref-list-1

This article has been cited by 2 HighWire-hosted articles: http://nn.neurology.org/content/3/6/e300.full.html\#\#otherarticles

This article, along with others on similar topics, appears in the following collection(s):

\section{All Cognitive Disorders/Dementia}

http://nn.neurology.org//cgi/collection/all_cognitive_disorders_dementi a

All Immunology

http://nn.neurology.org//cgi/collection/all_immunology

\section{HIV}

http://nn.neurology.org//cgi/collection/hiv

\section{HIV dementia}

http://nn.neurology.org//cgi/collection/hiv_dementia

Viral infections

http://nn.neurology.org//cgi/collection/viral_infections

Information about reproducing this article in parts (figures,tables) or in its entirety can be found online at:

http://nn.neurology.org/misc/about.xhtml\#permissions

Information about ordering reprints can be found online: http://nn.neurology.org/misc/addir.xhtml\#reprintsus

Neurol Neuroimmunol Neuroinflamm is an official journal of the American Academy of Neurology.

Published since April 2014, it is an open-access, online-only, continuous publication journal. Copyright $\odot$ 2016 American Academy of Neurology. All rights reserved. Online ISSN: 2332-7812.

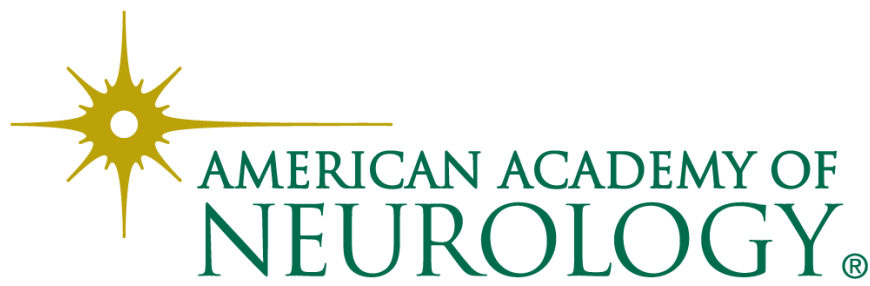

